\title{
ALLERGIC REACTION TO PATENT BLUE IN BREAST SURGERY: CASE REPORT
}

Elisa Rosa de Carvalho Gonçalves Nunes Galvão1, Danilo Rafael da Silva Fontinele², Antonio Fortes de Padua Filho', Pablo Francisco Diogo Lopes ${ }^{1}$

${ }^{1}$ Hospital São Marcos - Teresina (PI), Brazil.

¿Universidade Estadual do Piauí - Teresina (PI), Brazil.

Introduction: The evaluation of the sentinel lymph node in the surgical treatment of initial breast cancer has been widely used. To identify the lymph node, patent blue dye or technetium radiopharmaceutical can be used. However, there have been reports of IgE-mediated hypersensitivity reactions to the blue patent dye (mean incidence of 1.8\%), in some cases, severe, with severe hemodynamic repercussions that require vasoactive drugs. This frequency is higher than that of hypersensitivity reactions observed during anesthetic procedures. Therefore, we reported a case of allergic reaction to patent blue in the intraoperative period after periareolar subcutaneous injection. Case description: Female, 56-year old patient. Four months before, she presented with pleomorphic grouped calcifications in the upper quadrants of the left breast (Category 4) in mammography and ultrasound, increasing in the past 2 years. A stereotactic biopsy was performed, and showed a lesion compatible with carcinoma in situ, G3, G3 nuclear grade. She underwent a segmental resection of the left breast, with search for sentinel lymph node under general anesthesia induced by fentanyl, propofol and atracurium, maintained with sevoflurane, $50 \%$ of $\mathrm{O} 2$ and $50 \%$ of Ar. About 15 minutes after the patent blue injection, the patient had sudden tachycardia (HR=122bmp) and arterial hypotension (BP 80x40), simultaneously with the appearance of erythematous papules with bluish core in the trunk, abdomen and limbs. She was treated with volume replacement $(500 \mathrm{ml}$ of Ringer's Lactate solution) and vasopressor (Ephedrine, 15 micrograms). Ranitidine, diphenhydramine and hydrocortisone were administered. She was hemodynamically stable. Five hours after the end of the surgery, she was on complete remission. The anatomopathological examination showed carcinoma in situ, sentinel lymph node and free margins, tumor measuring $1.5 \times 0.6$ $\mathrm{cm}$. Immunohistochemical test showed invasive ductal carcinoma with extensive intraductal component, $80 \% \mathrm{ER}$, negative HER-2, and 40\% ki67 - Luminal B. She is still undergoing radiotherapy and hormone therapy. Conclusions: The reaction was manifested by tachycardia, hypotension and skin changes. She presented good response to volume replacement and vasopressor, and was in full remission after 5 hours 